\title{
COVID-19 Infection in Elderly, Adverse Outcomes and Annihilation of the Longevity Dream
}

\section{Vinod Nikhra*}

Department of Medicine, Hindu Rao Hospital \& NDMC Medical College, India

\section{Review Article}

Volume 2 Issue 1

Received Date: May 09, 2020

*Corresponding author: Vinod Nikhra, Senior Consultant and Faculty, Department of Medicine, Hindu Rao Hospital and NDMC Medical College, New Delhi, India, Email: drvinodnikhra@gmail.com
Published Date: May 27, 2020

DOI: $10.23880 /$ aii-16000115

\section{Abstract}

The SARS-CoV-2 Infection in Older Adults: The Covid-19 pandemic is impacting the elderly people in drastic ways. As a novel disease, it often presents with higher morbidity and adverse outcomes including mortality in the elderly. As a special age group, they face a significantly high risk for contracting the infection and developing severe illness due to physiological changes with aging and potential underlying health conditions.

Clinical Spectrum and Course of COVID-19: The clinical spectrum of COVID-19 varies from asymptomatic or paucisymptomatic presentation to a moderate to severe state characterized by respiratory failure necessitating mechanical ventilation and ICU support. Further, those developing critical clinical condition manifest complications like sepsis, septic shock, and multiple organ dysfunction syndromes. The elderly people are more likely to develop a severe and critical illness as compared to the younger age groups.

The Host Factors for COVID-19 in Elderly: In general, the elderly men display a higher mortality than women, especially if there are one or more underlying chronic health conditions. The age-related decline in physiological functions, attributable to reduced organ reserve and gradual functional decline in various organs in the elderly, is the key factor. In addition, the aging associated alterations in the immune response, the immune senescence accompanied by subclinical pro-inflammatory state and inflamm-aging, expose the elderly to higher infectivity and a severe form of illness. There is significantly reduced ACE2 expression with aging in the elderly age group in both genders, which is further reduced in the elderly with hypertension, heart disease and diabetes. The SARS-CoV-2 binding to ACE2 may exaggerate the pro inflammatory milieu, predisposing this age group with the reduced ACE2 expression, to greater disease severity and mortality. There are certain socio-economic reasons, as well. The elderly people are prone to experience a drop in the quality of life and neglected healthcare, which compromise their health in general and when exposed to Covid-19.

\section{Conclusion}

Annihilation of Longevity Dream: The aging is a complex process and affects virtually all organs of the body. A vast body of knowledge can now explain the changes that take place with aging at molecular and cellular level. The possibility of aging slowly and living longer, and lengthy-healthy life is alluring. As the life expectancy has increased with the improved lifestyle, progress in healthcare and technology have made possible to slow aging and achieve significant longevity. But, the high morbidity and mortality in the elderly with Covid-19 shows us that aging weakens the physiological systems and compromises function of various organs especially in those with the underlying conditions like diabetes and heart disease. The older adults or elderly age group, in light of its vulnerability to Covid-19 associated infectivity, disease severity, unfavorable prognosis and worsened mortality, now face the loss of the longevity dream.

Keywords: Covid-19; Cytokine Storm; Elderly Age Group; Immunosenescence; Inflamm-Aging; Multi Organ Dysfunction; ARDS; Organ Reserve and Redundancy; Longevity Research; SARS-CoV-2 
Abbreviations: MODS: Multiple Organ Dysfunction Syndromes; ARDS: Acute Respiratory Distress Syndrome; MOD: Multiple Organ Dysfunction; CRS: Cytokine Release Syndrome; SARS: Severe Acute Respiratory Syndrome; RLR: Rig-I-Like Receptor; MDA-5: Melanoma DifferentiationAssociated Protein 5; ER: Endoplasmic Reticulum; UPR: Unfolded Protein Response; IL: Like Interleukin; STAT3: Signal Transducer And Activator Of Transcription 3; NLR: Neutrophil-To-Lymphocyte Ratio; ACE2: AngiotensinConverting Enzyme 2; RAS: Renin-Angiotensin System; CR: Caloric Restriction.

\section{The COVID-19 Infection in Elderly}

\section{The Clinical Disease in Elderly}

The Covid-19 is a novel disease with variable clinical presentations. For the most of those infected with SARS$\mathrm{CoV}-2$, the disease may pass on as a non-event with few mild symptoms and recovery without any significant illness. Those with significant but moderate disease, the hospital admission with supportive treatment without need for assisted ventilation may suffice. In those with severe disease, the SARS-CoV-2 infection damages the alveolar tissue and triggers an overreaction of the immune system leading to hyper inflammation and cytokine storm. The variable spectrum of disease severity attended by variable course of the disease in an individual patient may make the therapeutic decisions difficult and requires a close and intense monitoring. There are, though, certain clear risk factors, including the age and underlying chronic health conditions, which foretell adverse outcomes for the Covid-19 in older adults or elderly age group [1].

As per the United Nations projections, by 2050, there will be more than twice as many people over 65 as there are children under 5 , and the number of people 65 years of age or older globally will surpass the number of people 15 to 24 years of age [2]. The global aging trends are likely to create widespread public health challenges, dramatically increasing the burden of non-communicable diseases as well as exposing the vulnerability to infectious diseases like Covid-19. Protecting the elderly population will become a major issue in maintaining global health.

\section{Steps in Progression of COVID-19}

Once inside the airways, the $S$ protein on the SARS-CoV-2 viral surface recognize and stick to the ACE2 receptors, followed by the virus infecting the ACE2-bearing cells lining the upper as well as the lower respiratory tracts and with the dying cells sloughing down and filling the airways the virus is carried deeper into the lungs as the thin layer of surfactant coating the airways becomes even thinner and the brush border less efficient to defend from the invading virus. The virus is able to transmit while still confined to the upper airways, before invading the lower respiratory tract and lungs and causing symptoms. In addition, the virus is able to infect ACE2-bearing cells in other organs, including the blood vessels, gastrointestinal tract, and kidneys. With the viral infestation, the activated immune system leads to inflammation and pyrexia, and in extreme cases, may damage the host tissues and organs. The blood vessels become inflamed and leaky, leading to pulmonary edema. The immune hyper activation is accompanied by the excessive cytokine release, during which, the immune system apart from damaging the host tissues, leads to increased susceptibility to infectious bacteria. The storms can also affect other organs besides the lungs, especially in those suffering chronic diseases.

\section{The Clinical Spectrum of Covid-19}

The clinical spectrum of COVID-19 (Figure 1) varies from asymptomatic or pauci-symptomatic presentation (Stage I) to moderate to severe states (Stage II a and IIb) characterized by respiratory failure necessitating mechanical ventilation and ICU support and those manifesting critical clinical condition (Stage III) with complications like sepsis, septic shock, and multiple organ dysfunction syndromes (MODS). The mild to moderate disease occurs in approximately in $81 \%$ of cases. The severe disease with dyspnoea and acute respiratory distress syndrome (ARDS) and lung infiltrates appearing within 48 hours occurs in $14 \%$ of cases. Whereas, the critical COVID-19 illness accompanied by respiratory failure, septic shock, and multiple organ dysfunction (MOD) or failure (MOF) has been seen in approximately $5 \%$ of cases [3].

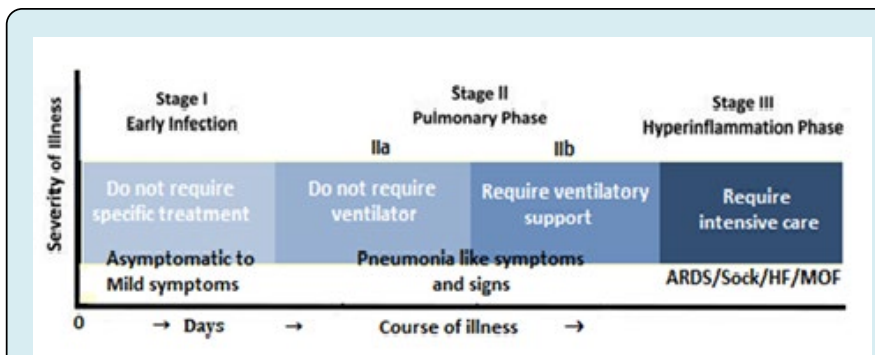

Figure 1: The spectrum and clinical stages of Covid-19.

The transition from milder symptoms to severe disease and ARDS has been related to an uncontrolled cytokine release by the hyperactive immune response. In patients who are going to have the worst outcomes with COVID-19 infections, the immune system becomes overactive with excessive stimulation of $\mathrm{T}$ cells and macrophages, resulting in cytokine storm with release of a large amount of pro 
inflammatory cytokines including interleukins (IL)-1, 6, 12 and 18. The excessive or uncontrolled levels of cytokines released, further activate more immune cells, resulting in hyper inflammation and cytokine release syndrome (CRS).

\section{The Morbidity and mortality in Elderly}

The Covid-19 pandemic is impacting the elderly people in drastic ways. As a special age group, they face a significantly high risk for contracting the infection and developing severe illness due to physiological alterations with aging and potential underlying health conditions. As per WHO, over $95 \%$ of the deaths occurred are in those aged 60 years or older. More than $50 \%$ of all fatalities involved people aged 80 years or older. Further, 8 out of 10 deaths due to Covid-19 occur in individuals with comorbidity, such as cardiovascular disease, hypertension, and diabetes and other underlying diseases [4]. As per the report posted on 31 March 2020 by the CDC, based on 7162 COVID-19 patients about whom the CDC had complete information, $37.6 \%$ had one or more underlying health condition. Further, out of Covid-19 ICU patients, $32 \%$ had diabetes, $29 \%$ heart disease, $21 \%$ chronic lung disease, and 9\% had compromised immune systems [5].

As compared to Covid-19, the earlier human Coronavirus disease outbreaks, the Severe Acute Respiratory Syndrome (SARS), which occurred in 2002-2003, registered a mortality rate of $9 \%$. The rate was much higher in elderly individuals, with the fatality rates approaching 50\% in individuals over 60 years of age [6]. Another novel human CoV, named Middle East Respiratory Syndrome-CoV (MERS-CoV), was found to be the causative agent in a series of highly pathogenic respiratory tract infections in Saudi Arabia and other countries in the Middle East, which emerged in the Middle East in 2012. Like, the SARS, the MERS outbreak was also associated with a higher mortality in the elderly age group [7].

\section{The Host Factors in Older Adults}

The Covid-19 is a novel disease, but the available studies point to a genetic factor making certain persons or a genetic stock more susceptible. In general, the men display a higher mortality than women, which could be due to one or more underlying health risk factors, such as smoking and drinking. In addition, women appear to have a stronger immune system than men. There are individual differences in immune response, which may lead one person to have severe disease [8]. The physiological changes associated with aging, decreased immune function and multimorbidity lead the older adults to high infectivity, disease severity and adverse outcome from Covid-19. It is becoming clear that the health status, in general, plays a crucial role in prognosis from Covid-19. People aging healthily are at less risk, compared to those harboring poor health and compromised physical fitness.

There are underlying reasons for the elderly people's greater susceptibility to Covid-19. The pre-existing chronic illnesses mark declining immunity and biological aging, which is more important than the chronological age. In addition, other factors like individual genetic make-up, the amount of virus load, the other microbes in the body including gut microbiota may play a role in acquirement of the infection and its progression.

\section{The Aging Process and Covid-19}

The age is an important epidemiological factor and the elderly people are at risk of severe infections possibly because the inefficient initial anti-viral immune response. The presence of the chronic diseases marks declining immunity and biological aging, which is more important than the chronological age. Due to various factors, the clinical severity and outcomes including the mortality from Covid-19 in elderly patients is higher than that in young and middleaged patients [9]. The elderly patients are more susceptible to severe illness and more likely to need admission to ICU than the younger patients [10]. Further, among the admitted patients, the incidence of ARDS, and MOD and MOF was higher in the elderly age group than that in the young and middle-aged groups. Furthermore, the response to drugs including Lopinavir and Ritonavir, supportive care, oxygen therapy and mechanical ventilation was statistically less effective in the elderly than those young and middle-aged.

In terms of laboratory tests, the proportion of elderly patients with an increased number of total leucocyte count and neutrophils in was significantly higher than the young and middle-aged groups, suggesting that elderly SARS-CoV-2 infected patients are more likely to suffer from a bacterial infection. In addition, the decrease in lymphocytes is delayed in the elderly patients, compared to the young and middleaged patients. This may be due to delayed recovery in the elderly due to asthenia including muscle atrophy and agerelated changes in the lungs compromising the physiological functions of the respiratory system and reduced airway clearance, reduced lung reserve, and reduced defence barrier function [11]. The C-reactive protein levels in elderly patients are significantly higher than that in the young and middle-aged groups. In context of imaging, the incidence of multilobe lesions in elderly patients is significantly higher than in young and middle-aged patients [12].

Finally, the lethality of Covid-19 infection in the elderly is significant and the age could be held as an independent risk factor for severity of disease and associated mortality. As reported by Verity et al, the age-related case fatality for 
Covid-19, age-group-wise was-for less than 30 years $\sim 0.06 \%$, for those $30-39$ years $0.14 \%, 40-49$ years $0.29 \%$ and for 50 59 years $1.2 \%$. It rose significantly for elderly to $3.9 \%$ in the $60-69$ age group, $8.6 \%$ to those $70-79$, and $13.4 \%$. For those 80 and over [13]. In the US, the CDC has reported that during 12 February to 16 March 2020, the adults older than 65 , were $31 \%$ of total cases, $45 \%$ of hospitalizations, $53 \%$ of ICU admissions, and $80 \%$ of all deaths associated with Covid-19, whereas those aged $\geq 85$ years showed the highest percentage of severe outcomes [14].

As much as $63 \%$ the deaths due to Covid-19 have been reported among the people who were over 60 years, while 60 and above age group formed $19 \%$ of all cases, as reported by Ministry of Health, Government of India. Further, 86\% deaths were associated with comorbidity related to diabetes, chronic kidney disease, hypertension, and heart disease. Compared to this, 47\% were infected with the virus in age group below 40 years age with death rate $7 \%$ and $34 \%$ infected between 40 to 60 years age group with $30 \%$ deaths [15].

\section{The Biological aging and its Fallouts}

The organ reserve is the ability of an organ to endure recurring stressful conditions and restore the homeostasis and normal physiological functions following such conditions [16]. A part of the age-related decline in physiological functions is attributable to reduction in organ reserve as present in various body systems and the gradual functional decline in specific tissues or organs (e.g., immune-, musculoskeletal-, nervous systems) is a key characteristic of aging. Although organs vary in the rate of functional decline with age, the gradual linear decline of reserve capacity with age shows values ranging from $0.5 \%$ to $1.4 \%$ per year [17]. The decline appears to accelerate by the fifth decade of age, which may explain, in part, the age-related increase in vulnerability to disease and disability (Figure 2).

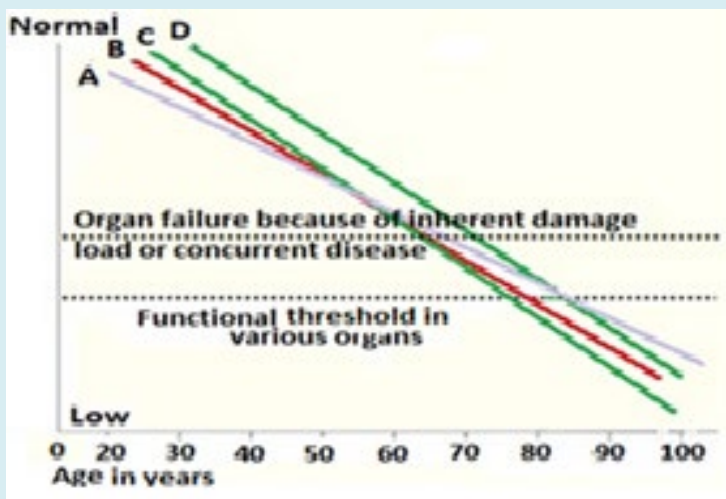

Figure 2: Aging and Functional Threshold and Reserve of Various organs.
On the cellular level, several metabolic pathways exhibit excess metabolic capacities, such as, bioenergetics pathways and antioxidants system. The excess metabolic capacities can be viewed as an adaptability mechanism that substantiates organ reserve and contributes to the cellular defence systems. With aging, the metabolic excess capacities or organ reserves are impaired or exhausted; the ability to cope with stress is reduced leading to cell senescence, transformation, or apoptosis [18]. The decrease in organ mass, tissue anatomy and physiological function with age have been demonstrated in the human heart, brain, liver, kidney, salivary glands, stomach, and muscle tissue. The major storage reservoir of protein in the body is skeletal muscle, which tend to be lost with age manifesting as decrease in muscle mass and strength. By $6^{\text {th }}$ decade of life, voluntary muscle contractile strength is decreased by over $20 \%$ in both men and women. By the time men and women are in their 7 th or 8th decade of life, on average they have lost about $20 \%$ to $40 \%$ of the contractile strength of voluntary muscles, and over $50 \%$ by the $9^{\text {th }}$ decade. The rate and severity of decline varies between individuals and even between specific organs within individuals and may not always correlate with age. Further, the clinical trials have shown that declines in organ mass and function are reversible in some tissues, such as muscle and brain.

Apart from, the effacement of the excess metabolic capacities, aging at the molecular level is characterized by the progressive accumulation of molecular damage through environmental and metabolically generated free radicals, spontaneous errors in biochemical reactions and nutritional components. The damage to the maintenance and repair pathways comprising homeo-dynamic mechanisms leads to age-related failure of homeo-dynamics, increased molecular heterogeneity, reduced stress tolerance, altered cellular functioning, senescence, and apoptosis [19]. These factors compromise the bioenergetics reserve capacity and accelerate the process of aging and increase the risk of age-related disorders. With age, the functional reserve and redundancy of various organs wear out. Most organ functions decline by about one percent per year, the decline starting at about age forty or earlier, continue throughout life often without perceptible loss of function. Further, the age of functional decline is variable for different organs in different individuals. Nevertheless, the internal organs including brain, heart, lungs, and kidneys show a slow and gradual decline. The older adults often have multiple age-related functional impairments at level of various organs due to loss of organ reserve and redundancy.

\section{Concepts of Genome Stability and Aging}

Patho physiologically, a number of diverse stimuli induce senescence. These factors appear to converge on 
certain pathways that influence cell cycle regulation, DNA repair and apoptosis, and the process of cellular senescence. At the cellular level, these pathways are regulated by the tumor suppressor proteins p53 and pRb. The p53 is a crucial mediator of the cellular response to damaged DNA and dysfunctional telomeres, and in turn activates the cyclindependent inhibitor p21. It is considered that senescence occurs via the p53 pathway in response to DNA damage and telomere attrition, whereas the $\mathrm{p} 16 / \mathrm{pRb}$ pathway mediates senescence caused by oncogenic stimuli, chromatin disruption, and other cellular stresses [20]. In addition, the chronic oxidative stress compromises telomere integrity and accelerates the onset of senescence in animal studies as well as in the human endothelial cells (Figure 3).

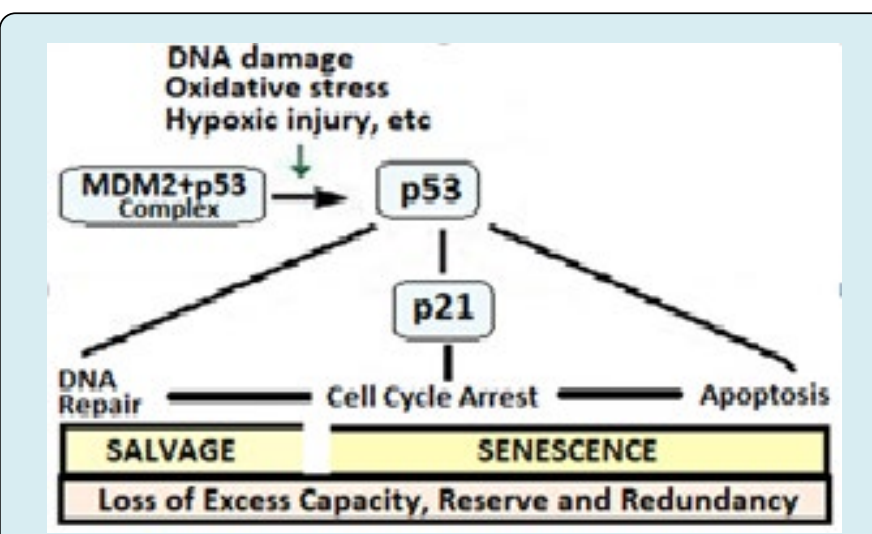

Figure 3: The Concept of Genome Stability and Aging.

\section{The Bio-Homeo-Dynamic space}

The living systems owe their survival and health to a series of complex biochemical pathways of maintenance and repair. Simultaneously, the defence systems create the homeodynamic space characterized by stress tolerance, molecular damage control and continuous remodeling. Further, the biological systems maintain the biological rhythms, sense, and respond to intra and extra-cellular stressors through innate and adaptive immune responses, scavenge and remove of reactive oxygen and other free radical species and keep thermal regulation and neuro-endocrine balance [21]. The aging and the age-related diseases can be held as the consequences of a progressive shrinkage of the homeodynamic space, due to the failure of maintenance and repair. Aging is a complex process due to the interaction of many lifelong influences which include heredity, environment, culture, diet, exercise and leisure, past illnesses, and various other factors.

The aging organs slowly lose function, which may not be noticeable because the organs are rarely used to their fullest ability and have a reserve ability to function beyond the usual needs. Some body systems begin aging as early as age 30, whereas other aging processes are not common until much later in life. Further, age affects each individual differently from others. The cell membrane changes affect diffusion of oxygen and nutrients and removal carbon dioxide and other wastes. With aging, the connective tissue becomes stiffer making the organs, blood vessels and airways more rigid.

\section{Senescence, Debility and Age-related Diseases}

The major changes in organ reserve occur in the heart, lungs, and kidneys, usually appearing slowly and over a long period. The stressors producing an extra workload include illness, significant life changes and sudden increased physical demands on the body as a result of change in activity level. The recovery from illnesses is seldom complete, leaving some residual disability. The loss of reserve also makes it harder to restore homeostasis in the body with robustness.

The process of aging of organs is universal, progressive, and vastly irreversible. Some individuals may age without much deterioration in body organs and systems, whereas others may be ridden with the extreme age-associated changes. Further, the aging process may affect some body systems more severely whereas others are spared from a serious disability. Thus, aging is associated with a progressive but varying decline in numerous physiological functions and significantly affects the body organs and various systems including the lungs, heart, and vasculature, kidneys and brain.

\section{The Immune Response in Covid-19}

The high risk to Covid-19 in the elderly is due, in part, to the Immunosenescence which encompasses the changes in the immune system with age. The immune system has two sets of defence against viruses and other pathogens. The first response is, in form of naïve leukocytes that attack invading microbes within minutes to hours. The second line of response is, in form of precisely targeted antibodies and $\mathrm{T}$ cells that surge later over several days.

\section{The Immunosenescence and Inflamm-aging}

With advancing age, the body has fewer naive $\mathrm{T}$ cells that have not yet been programmed to defend against a specific microbe. Whereas, the body does retain the memory T cells another age-related change is the natural killer cells do not work efficiently, responding too late and too little. Similarly, the B cells in the elderly do not produce enough antibodies (Figure 4). In general, there is a progressive age-related decline of innate and adaptive immune responses [22]. 
In addition, the age-associated chronic diseases such as heart disease, metabolic including diabetes, autoimmune diseases, and malignancy, along with the effects of their treatments on general health, substantially affect responses to infectious diseases, both bacterial as well as viral [23].

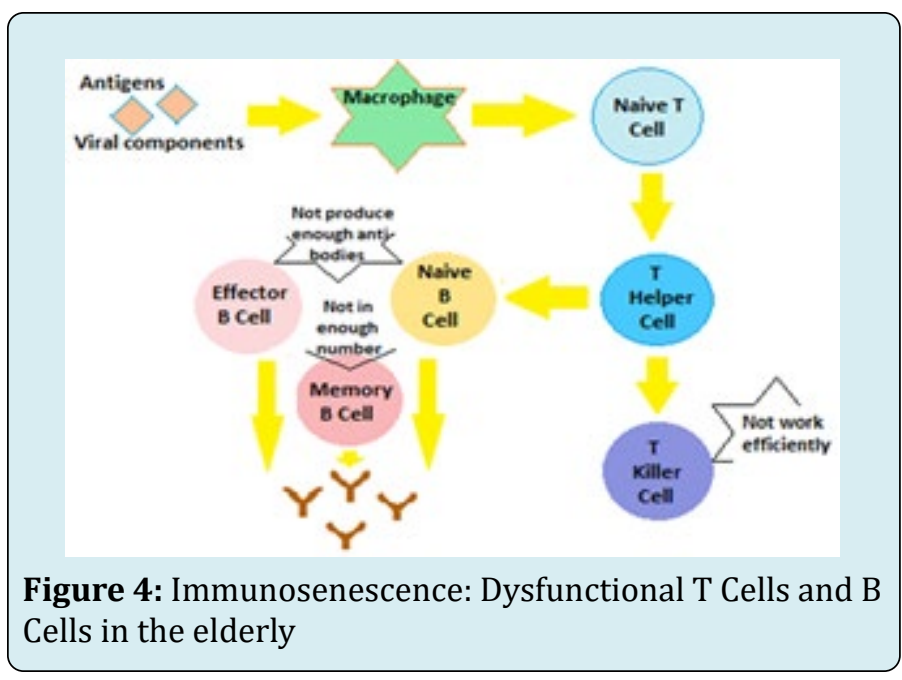

The Immunosenescence is accompanied by subclinical accumulation of pro-inflammatory factors and inflammaging [24]. The Immunosenescence and inflamm-aging, acting in concert, mutually influence each other and lead to a decreased adaptive immune response, which reinforces the stimulation of the innate immune response. Thus, the innate immune response is conserved and the immune cells are constantly maintained in an alert state due to chronic lowgrade inflammation, which is a physiological response to the life-long antigenic stress but without the essential counterregulation by anti-inflammatory molecules as seen with aging.

The adaptive immune system is composed of the cellular and the humoral immune response. The cellular response includes CD4+ (helper) and CD8+ (memory) T cell populations. With aging, there is an increase in the number of CD8+ T memory cells and, later on, that of B cells due to a continuous chronic antigenic stimulation. The chronic antigenic stimulation, thus, leads to the phenomenon of inflamm-aging and immune exhaustion, characterized by the emergence of inhibitory receptors, such as PD-1 and CTLA-4. The CD4+ T cell population also undergoes similar changes to CD8+ T cells and there is an increase in Treg population as well as the pro-inflammatory Th17 subpopulation also increases with aging. Finally, the B cell compartment is also altered with aging. The clonal expansion, cytokine production, and specific antibody production are compromised leading to increased incidence of infections, chronic diseases, and cancer in the elderly [25]. The changes affect the innate immune system, which overtakes the altered adaptive immune system.

The cumulative changes in critical B- and T-cell subpopulations, from natural killer cells to the activated $\mathrm{T}$ cells and $\mathrm{B}$ cells, and innate immune system lead to increased likelihood of infection and bringing about a pro-inflammatory state. With aging, there are defects in monocytes/macrophages function and expression of proinflammatory cytokines such as interleukin (IL)-1, IL-6, and tumor necrosis factor (TNF)- $\alpha$, and anti-inflammatory cytokine IL-10 by these cells after stimulation with lipopolysaccharide (LPS) and IFN- $\gamma$. Aging seems to affect the immune system both quantitatively as well as qualitatively, leading to deregulated response to infection, manifesting as exaggerated inflammation and excessive tissue damage.

\section{The Host Cell Immune Response}

In general, the host cells respond to the virus infection by recruiting an innate antiviral response to limit the spread of the infection and resorting to induce an adaptive immune response to eventually clear the virus. In the case of SARS-CoV-2, the innate immune system is triggered by recognizing dsRNA and $5^{\prime}$-triphosphate-bearing RNA molecules arising as replication intermediates in the cytosol by the intracellular sensors of the Rig-I-like receptor (RLR) family, such as retinoic acid-inducible gene 1 (RIG-I) and melanoma differentiation-associated protein 5 (MDA5) which are expressed in various host cells [26,27]. The toll-like receptors (TLRs) which are expressed on the cell surface or reside in the endosomes of immune cells can also recognize CoVs nucleic acids or proteins. This is followed by the expression of IFN- $\beta$, secretion of Type-I IFN and proinflammatory cytokines, and an inflammasome response resulting in an antiviral state of the infected cells. In addition, the viral infection and replication are associated with the endoplasmic reticulum (ER) stress. The ER can sustain a high load of protein content without being overwhelmed. However, when the ER's capacity for folding and processing proteins is exceeded, unfolded or misfolded proteins rapidly accumulate in the lumen and the ER stress response is induced. The ER stress leads to activation of Unfolded Protein Response (UPR) pathways eventually triggering apoptosis, which is utilized by the host cells to inhibit viral replication.

\section{Immunological Basis of Severe Covid-19}

There is a complexity of the virus-host interactions that occur within the cell, tissues, or the whole body. The SARSCoV-infected peripheral blood mononuclear cells lead to the up regulation of expression of various cytokines, including IL-8 and IL-17, and the activation of macrophages and the 
coagulation pathways. The protein kinases, the key regulators in signal transduction, control a wide variety of cellular processes. They are linked to cellular immune responses, like interleukin (IL) signaling, IL-6 and -8, and influence the $\mathrm{CoV}$ infection and CoV-induced inflammation. In general, the difference in the immune and inflammatory responses determines the outcome of the infection and responsible for the differential activation of the Signal transducer and activator of transcription 3 (STAT3) pathway, involved in lung inflammation and cellular repair [28].

The pyroptosis is a inflammatory form of programmed cell death and appears to be the possible mechanism for the increased virulence of SARS-CoV-2. The SARS-CoV Viroporin 3a triggers the activation of the NLRP3 inflammasome and the secretion of IL- $1 \beta$ by macrophages, leading to SARS$\mathrm{CoV}-2$ induced cell pyroptosis46. The patients have increased IL-1 $\beta$ in the serum, an indicator of the pyroptosis. The severe SARS-CoV-2 infection is likely to cause cell pyroptosis, especially in lymphocytes, through the activation of NLRP3 inflammasome. The response is an overdrive, in form of a fusillade of inflammatory molecules called cytokines, which attack the virus infested lungs, leading to a hyper inflammatory state, which is a harbinger of ARDS, a common cause of Covid-19 deaths.

The cytokine fusillade, however, varies by gender, with the older men having more cytokine-producing cells and thus faring worse than older women [29]. In the severely ill SARS-CoV-2 patients, the disease may progress rapidly from viral pneumonia to acute respiratory failure. The neutrophilto-lymphocyte ratio (NLR) has been identified as an independent risk factor for severe illness in these patients. The elderly patients with NLR $\geq 3.13$ are more likely to develop severe pneumonia and ARDS and should have rapid access to intensive care unit and respiratory support [30]. A number of elderly people dying from Covid-19 die because of complications related to the coexisting chronic diseases without developing pneumonia or ARDS. Further, the Immunosenescence leaves older people who have survived Covid-19, without robust immunity, if they be exposed to the virus again. The associated chronic conditions like CVD, lung disease, diabetes, or kidney disease also weaken the body's immunity. The elderly people having a weaker immune memory than the young people are more vulnerable to the disease.

\section{The ACE2 Homeostasis and Covid-19}

\section{Angiotensin-converting enzyme 2 (ACE2)}

The ACE2, or ACEH (ACE homolog), is an integral membrane protein and a zinc metalloprotease of the ACE family. The human ACE2 protein sequence consists of 805 amino acids, including a N-terminal signal peptide, a single catalytic domain, a C-terminal membrane anchor and a short cytoplasmic tail. ACE2 cleaves angiotensin I and II as a carboxypeptidase. Functionally, there are two forms of ACE2. The membrane-bound ACE2 contains a Tran's membrane domain and an extracellular domain, which is used by SARS$\mathrm{CoV}-2$ as the receptor. The other, soluble form of ACE2 lacks the membrane anchor and circulates in small amounts in the blood.

There is rich ACE2 surface expression in nasal mucosa and nasopharynx, oral mucosa and tongue, respiratory tracts, and lung alveolar epithelial cells. In addition, there is an abundant presence of ACE2 on arterial and venous endothelial cells, and arterial smooth muscle cells and oesophageal upper and stratified epithelial cells, absorptive enterocytes from ileum and colon, cholangiocytes, myocardial cells, renal proximal tubule cells, urinary bladder and urothelial cells, and skin, lymph nodes and brain. The abundant ACE2 expression has been linked with the pathogenesis involving vasculitis, deranged immune function, extensive pulmonary inflammation, and diffuse alveolar damage with hyaline membrane formation, and the severe clinical manifestations and the multi-organ dysfunction observed Covid-19 in patients [31]. It appears that apart from gaining its entry through the ACE2, the SARS-CoV-2 subsequently downregulates the ACE2 expression leading to loss of its protective effects in various organs including lungs and the vasculature, which may have significant impact on the pathogenesis of the disease.

\section{Interaction between ACE2 and Covid-19}

The epidemiological studies have documented that different sex and age groups have different susceptibility to SARS-CoV-2 infection, which may be linked to ACE2 expression. There is significantly reduced ACE2 expression with aging in the elderly age group in both genders. In animal studies, a higher ACE2 content was noticed in old female than male, which may explain comparatively worse prognosis of Covid-19 in elderly men [32]. In addition, the elderly, especially those with hypertension and diabetes, have reduced ACE2 expression and up regulation of angiotensin II proinflammatory signaling. The SARS-CoV-2 binding to ACE2 may exaggerate this proinflammatory milieu, predisposing the elderly people to greater disease severity and mortality. The ACE2 is predominantly expressed in surfactant-secreting type II alveolar cells in lungs, bronchiolar epithelium, and endothelium and smooth muscle cells of pulmonary vessels. Further, it is more abundantly expressed in the apical than the basolateral areas of lungs. Furthermore, the ACE2 expression is proportionately correlated to the epithelial differentiation of the alveolar tissue. The undifferentiated cells poorly express ACE2, while well-differentiated cells richly express 
ACE2. The studies indicate that infection of human airway epithelia by SARS-CoVs correlates with the state of cell differentiation and ACE2 expression and localization [33].

The lungs appear to be the most vulnerable target organs in Covid-19, because of the vast surface area making the lung highly susceptible to inhaled viruses. About 83 percent of ACE2-expressing cells are alveolar type II cells, which are exposed and can serve as a reservoir for the viral invasion. The ACE2 is not only the entry receptor of the virus but also protects from lung injury and the angiotensin-(1-7) generated by degradation of Ang II by ACE2, has vasoprotective functions. In addition, it appears that ACE2 competitively binds with SARS-CoV-2 not only to neutralize the virus but also rescue cellular ACE2 activity which negatively regulates the renin-angiotensin system (RAS) to protect the lung from injury [34]. The high lethality associated with SARS-CoV-2 infection could be because of dysregulation of the pulmonary protective mechanisms [35]. Whereas as demonstrated in mice experiments the SARS-CoV down regulates ACE2 protein by binding its spike protein, contributing to severe lung injury [36]. The decreased ACE2 availability may contribute to oxidative stress and subsequent lung injury.

\section{The RAAS Activity and Covid-19 Prognosis}

The perspective that ACE inhibitors, like ramipril and ARBs, like losartan may increase ACE2 expression, have raised concerns about their safety in patients with Covid-19. The interaction between the SARS-CoVs and ACE2 has been proposed as a potential factor in their infectivity by some researchers, and there are concerns about the use of these drugs may alter the ACE2 expression and attended by its fallouts [37]. Currently, there are insufficient data available to translate it into clinical practice. On the other hand, it is held that an abrupt withdrawal of these drugs in high-risk patients, having heart failure, coronary heart disease and hypertension may result in clinical instability and adverse outcomes. Further, despite substantial structural homology between ACE and ACE2, their enzyme active sites are distinct and the ACE inhibitors and ARBs in clinical use do not directly affect the ACE2 activity. Thus, it is advisable that an ACE inhibitor or ARB should be continued in known or likely Covid-19 patients with stable condition [38].

The SARS-CoV-2 utilizes and affects ACE in multiple ways. Apart from its entry through the ACE2, SARS-CoV-2 subsequently down-regulate ACE2 expression. Further, with the continued viral infection and replication contribute to reduced membrane ACE2 expression as the ACE2 sites are taken up and damaged by subsequent viral invasions. In addition, the SARS-CoV-2 binding to ACE2 exaggerates proinflammatory background in the elderly patients. The reduced ACE2 activity in the lungs results in unopposed angiotensin II accumulation and local RAAS activation, contributing further to inflammation and neutrophil infiltration and associated with high morbidity and mortality in the elderly patients with Covid-19.

\section{General health and Socioeconomic factors}

There are certain socio-economic reasons, as well. In many societies, elderly people are prone to live in poverty, experience a drop in the quality of life and neglected healthcare, which compromises their health in general [39]. Further, prioritizing younger patients in case of limited resources such as availability of ventilators and ICU beds, may exclude the elderly people, who already carry a poor prognosis, thus, still worsening their chances of survival [40]. Many of the elderly are able to receive only compassionate care, which means they may lose provision of the scarce resources and overburdened intensive care in favour of the younger Covid-19 patients having a better chance at survival [41].

\section{Conclusion: Annihilation of the Longevity Dream}

\section{The concepts in aging and longevity}

The aging is a complex process and affects virtually all organs of the body. A vast body of knowledge can now explain the changes that take place with aging at molecular and cellular level. There do exist possibilities of being able to reverse the aging process [42]. The possibility of a lengthyhealthy life is alluring. As the life expectancy at birth rises and there is taking place an improvement in average and maximum lifespan, the possibility of living life more than never seems logical. The science gives visions; the technology makes the visions possible. The future technology appears to offer us visions that rival the dreams of myth and legend. As per the Arthur C. Clarke's Third Law-'any sufficiently advanced technology is indistinguishable from magic' [43]. One of these magical dreams is that of exponential life extension.

The longevity research has always looked forward to retard aging of immune system, reverse stem cell aging and to keep alive or preserved through good health practices, nutritional supplements, caloric restriction (CR) and organ transplantation, and finally cryopreserved the human body after death till the advanced molecular repair technologies are available. The realization of dream of the state of nonaging to achieve immortality or eternal life was a far-fetched dream which dwelt on the possibility is that technology will be able to repair the damage done to our tissues with age and 
thus granting us longevity with good quality of life.

\section{Retarding aging and the longevity dream}

But the aging is universal in the kingdom of living. We find people aging; we ourselves age and grow older. There has evolved a whole novel understanding of the biology of aging. Thinking rationally, it is unlikely that something like a pill or potion can reverse the changes and dysfunction associated with aging. At the same time, the improved lifestyle, progress in healthcare and technology has made possible to slow aging, achieve significant longevity. There are established strategies aiming to reduce the oxidative stress which exacerbates aging process and leads to debility to human body in general and CVS in particular. The life-style changes in form of healthy diet, regular physical exercise other interventions like smoking cessation and intervention in sleep disorders are established practical ways to prevent metabolic diseases, CVDs, and neuro-degenerative disorders (Figure 5).

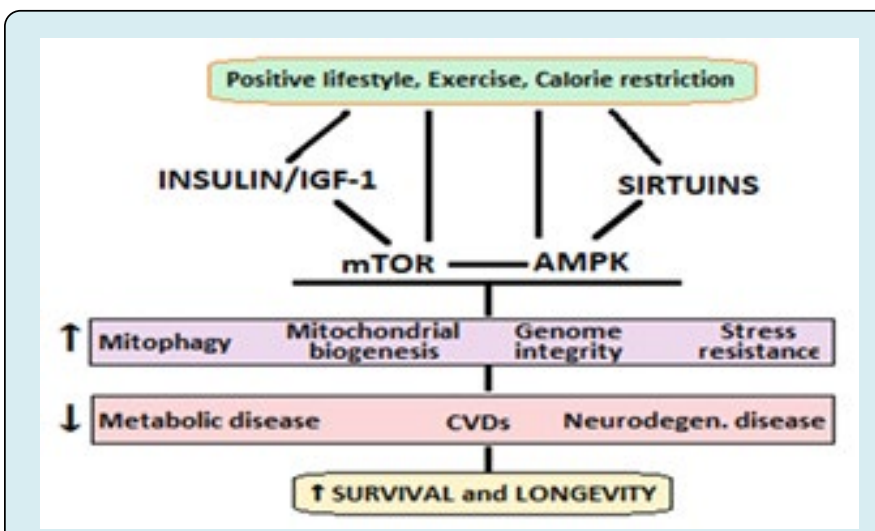

Figure 5: The synergistic pathways for disease protection and longevity

\section{Annihilation and loss of the longevity dream}

The high morbidity and mortality in the elderly with Covid-19 s shows us that aging weakens the physiological systems and body organs. The irrational hopes from the unproven concepts of longevity research move us away from terra-ferma and are detrimental to rational scientific behavior. The elderly apart from physiological alterations in the immunity and various organs frequently suffer with the underlying conditions like diabetes and heart disease, which increase the infectivity risk as well as pose them to unfavorable prognosis from Covid-19. Further, the lifetime exposure oxidants, various endocrine disruptors and air pollution exacerbate the aging related physiological deficits in the lungs, heart and vasculature and other organs and make them vulnerable to SARS-CoV-19 infection. The older adults or elderly age group, in light of their vulnerability to Covid-19 associated infectivity; disease severity and worsened mortality, now face the loss of the longevity dream.

\section{References}

1. Guo YR, Cao QD, Hong ZS, Tan YY, Chen SD, et al. (2020) The origin, transmission and clinical therapies on coronavirus disease 2019 (COVID-19) outbreak - An update on the status. Mil Med Res 7(1): 11.

2. World population prospects (2019) Department of Economic and Social Affairs Population Dynamics, United Nations.

3. McIntosh K, Hirsch MS, Bloom A (2020) Coronavirus disease 2019 (COVID-19): Epidemiology, virology, clinical features, diagnosis, and prevention.

4. (2020) World Health Organization Statement.

5. CDC COVID-19 Response Team (2020) Preliminary Estimates of the Prevalence of Selected Underlying Health Conditions Among Patients with Coronavirus Disease 2019-UnitedStates, February 12-March 28, 2020. Center of diseases control and Prevention 69(13): 382-386.

6. (2004) Cumulative Number of Reported Probable Cases of Severe Acute Respiratory Syndrome (SARS). World Health Organization.

7. (2019) Middle East respiratory syndrome coronavirus (MERS-CoV). World Health Organization.

8. Li JY, You Z, Wang Q, Zhou ZJ, Qiu Y, et al. (2020) The epidemic of 2019-novel-coronavirus (2019-nCoV) pneumonia and insights for emerging infectious diseases in the future. Microbes Infect 22(2): 80-85.

9. Liu K, Chen Y, Lin R, Han K (2020) Clinical features of COVID-19 in elderly patients: A comparison with young and middle-aged patients. J Infect 80(6): e14-e18.

10. Guan WJ, Ni ZY, Yu Hu, Liang WH, Ou CQ, et al. (2020) Clinical characteristics of 2019 novel coronavirus infection in China. N Eng J Med 382: 1708-1720.

11. Liu W, Tao ZW, Lei W, Yuan ML, Liu K, et al. (2020) Analysis of factors associated with disease outcomes in hospitalized patients with 2019 novel coronavirus disease. Chin Med J 133(9): 1032-1038.

12. Yang Y, Lu QB, Liu M, Wang Y, Zhang A, et al. (2020) Epidemiological and clinical features of the 2019 novel coronavirus outbreak in China. Med Rxiv. 
13. Verity R, Okell LC, Dorigatti I, Winskill P, Whittaker C, et al. (2020) Estimates of the severity of coronavirus disease 2019: a model-based analysis. Lancet Infectious Diseases 3099(20): 30243-30247.

14. CDC COVID-19 Response Team (2020) Severe Outcomes among Patients with Coronavirus Disease 2019 (COVID-19) - United States, February 12-March 16, 2020. Morb Mortal Wkly Rep 69(12): 343-346.

15. (2020) Ministry of Health and Family Welfare, Updates on COVID-19.

16. Neustadt J, Pieczenik S (2008) Organ reserve and healthy aging. Integrative Medicine 7(3): 50-52.

17. Sehl ME, Yates FE (2001) Kinetics of human aging I. Rates of senescence between ages 30 and 70 years in healthy people. J Gerontol A Biol Sci Med Sci 56(5): B198-208.

18. Atamna H, Tenore A, Lui F, Dhahbi JM (2018) Organ reserve, excess metabolic capacity, and aging. Biogerontology 19(2): 171-184.

19. Rattan SI (2008) Increased molecular damage and heterogeneity as the basis of aging. Biol Chem 389(3): 267-272.

20. Moslehi J, DePinho RA, Sahin E, Sinclair D, North B (2012) Telomeres and Mitochondria in the Aging Heart. Circ Res 110: 1226-1237.

21. Nikhra V (2019) The Concepts of Biorhythms, Redundancy and Reserve: Impact on Cardiovascular Aging and Disease. Cardiology 23(6): 226-235.

22. Poland GA, Ovsyannikova IG, Kennedy RB (2018) Personalized Vaccinology: A review. Vaccine 36(36): 5350-5357.

23. Koff WC, Williams MA (2020) Covid-19 and Immunity in Aging Populations-A New Research Agenda. N Eng J Med.

24. Fulop T, Larbi A, Dupuis G, Page AL, Frost EH, et al. (2018) Immunosenescence and Inflamm-Aging as Two Sides of the Same Coin: Friends or Foes?. Front Immunol 1960.

25. Fulop T, Dupuis G, Witkowski JM, Larbi A (2016) The role of Immunosenescence in the development of age-related diseases. Rev Invest Clin 68(2): 84-91.

26. Loo YM, Gale M (2011) Immune signaling by RIG-I-like receptors. Immunity 34(5): 680-692.

27. Lazarte JMS, Thompson KD, Jung TS (2019) Pattern Recognition by Melanoma Differentiation-Associated Gene 5 (Mda5) in Teleost Fish: A Review. Front Immunol
10: 906.

28. Selinger C, Tisoncik Go J, Menachery VD, Agnihothram S, Law GL, et al. (2018) Cytokine systems approach demonstrates differences in innate and pro-inflammatory host responses between genetically distinct MERS-CoV isolates. BMC Genom 15(1): 1161.

29. Marquez EJ, Chung CH, Marches R, Rossi RJ, Belaid DN, et al. (2020) Sexual dimorphism in human immune system aging. Nature Communications 11: 751.

30. Liu J, Liu Y, Xiang P, Lin P, Xiong H, et al. (2020) Neutrophilto-Lymphocyte Ratio Predicts Severe Illness Patients with 2019 Novel Coronavirus in the Early Stage. Preprint from medRxiv and bioRxiv.

31. Hamming I, Timens W, Bulthuis MLC, Lely AT, Goor HV (2004) Tissue distribution of ACE2 protein, the functional receptor for SARS coronavirus. A first step in understanding SARS pathogenesis. J Pathol. 203(2): 631637.

32. Xie X, Chen J, Wang X, Zhang F, Liu Y (2006) Age- and gender-related difference of ACE2 expression in rat lung. Life Sci 78(19): 2166-2171.

33. Jia HP, Look DC, Shi L, Hickey M, Pewe L, et al. (2005) ACE2 Receptor Expression and Severe Acute Respiratory Syndrome Coronavirus Infection Depend on Differentiation of Human Airway Epithelia. J Virol 79(23): 14614-14621.

34. Imai Y, Kuba K, Penninger JM (2005) The discovery of angiotensin-converting enzyme 2 and its role in acute lung injury in mice. Exp Physiol 93(5): 543-548.

35. Zhang H, Penninger JM, Li Y, Zhong N, Slutsky AS (2020) Angiotensin-converting enzyme 2 (ACE2) as a SARSCoV-2 receptor: molecular mechanisms and potential therapeutic target. Intensive Care Medicine 46(4): 586590.

36. Kuba K, Imai Y, Rao S, Gao H, Guo F, et al. (2005) A crucial role of angiotensin converting enzyme 2 (ACE2) in SARS coronavirus-induced lung injury. Nat Med 11(8): 875879.

37. Al Ghatrif M, Cingolani O, Lakatta EG (2020) The Dilemma of Coronavirus Disease 2019, Aging, and Cardiovascular Disease: Insights from Cardiovascular Aging Science. JAMA Cardiol.

38. Vaduganathan M, Vardeny O, Michel T, McMurray JJV, Pfeffer MA, et al. (2020) Renin-angiotensin-aldosterone system inhibitors in patients with Covid-19. N Engl J Med 382: 1653-1659. 
39. Kruk ME, Gage AD, Arsenault C, Jordan K, Leslie HH, et al. (2018) High-quality health systems in the Sustainable Development Goals era: time for a revolution. The Lancet Global Health 6(11): E1196-E1252.

40. (2020) 'Every ventilator becomes like gold'-doctors give emotional warnings from Italy's Coronavirus outbreak. World Economic Forum.
41. (2020) Agonizing decisions being made in Spain's virus hot spots.

42. Vinod N (2018) Future Projections and Fallouts of Exponential Longevity and Revival from Cryopreservation. Res Med Eng Sci 6(4): 000640.

43. Arthur CC (2000) Profiles of the future: An inquiry into the limits of the possible. Open Library. 